in both phases. The cellular, morphological, biochemical and biomechanical properties of the embryoid bodies and engineered tissues were analyzed.

Compared with normoxic conditions, cell differentiation in hypoxic conditions led to improved synthesis of collagens I and II (up to 2.9-fold and up to 3.4-fold, respectively) and glycosaminoglycans (up to 1.9-fold), thereby improving the biomechanical functionality of the cartilage. During the tissue engineering phase, normoxic conditions resulted in thicker and heavier constructs than those assembled in a hypoxic environment. Oxygen availability also altered the cellular expression of CD44, CD105 and platelet derived growth factor receptor $\alpha$, which the authors identified as potential markers for cartilage synthesis.

The discovery that hypoxic differentiation conditions boost the production of cartilage extracellular matrix and enhance the biomechanical function of cartilage constructs is an important step towards replacing damaged or diseased cartilage with tissue developed from stem cells, the authors conclude.

Original article Koay EJ and Athanasiou KA (2008) Hypoxic chondrogenic differentiation of human embryonic stem cells enhances cartilage protein synthesis and biomechanical functionality. Osteoarthritis Cartilage 16: 1450-1456

\section{New strategies have more impact than drug choice on improvements in RA management}

A Spanish cohort study of patients with rheumatoid arthritis (RA), conducted over a 4-year period during which novel therapies were introduced into clinical practice, has shown that the management of disease activity has generally improved in recent years. Surprisingly, however, the new drugs only partially account for this benefit. González-Alvaro et al. suggest that other factors - primarily changes to treatment strategies - have had a greater impact on routine patient care than the choice of therapeutic agent.

Improvements in outcomes were not as dramatic as those observed in clinical trials, but by the end of their study the median disease activity score had decreased. Furthermore, although disability and radiographic damage worsened, the decline in these outcomes was less than expected. The authors hypothesize that calendar year had a greater influence on these outcomes than treatments because of improved efficiency in treatment for RA.

At the study outset in 2000 , only $8(1.8 \%)$ of the cohort's 789 patients were receiving leflunomide or an anti-tumor necrosis factor agent, a number which had risen to 144 (31.9\%) by 2004. The proportion of patients receiving combination therapy also increased during this period, and the combinations themselves evolved to more often include biologic agents. Other research indicates that the dose of methotrexate administered to patients in Spain substantially increased during this time.

Original article González-Alvaro I et al. (2008) Trends towards an improved disease state in rheumatoid arthritis over time: influence of new therapies and changes in management approach: analysis of the EMECAR cohort. Arthritis Res Ther 10: R138

\section{Bortezomib improves arthritis in a mouse model}

The activity of nuclear factor $\kappa \mathrm{B}(\mathrm{NF} \kappa \mathrm{B})$, a key regulator of several proinflammatory cytokines, is controlled by an inhibitor, which, in turn, is negatively regulated by proteasomal degradation. Blocking this degradation might, therefore, have an anti-inflammatory effect by inhibiting NFkB activity. Lee et al. assessed the efficacy of the proteasome inhibitor bortezomib on collagen-induced arthritis ( $\mathrm{CIA})$, a mouse model of rheumatoid arthritis.

Two doses of type II collagen, administered 2 weeks apart, induced CIA in DBA/1 mice. Once arthritis was established (4 weeks after the initial injection), bortezomib was given intraperitoneally twice a week for 2 weeks at $0.01,0.1$ or $1.0 \mathrm{mg} / \mathrm{kg}$. Control (non-ClA) and untreated mice were given phosphatebuffered saline twice a week. Doses of 0.1 and $1 \mathrm{mg} / \mathrm{kg}$ bortezomib effectively reduced the mean arthritis score and paw thickness, and resulted in markedly less joint destruction (asseszsed by three-dimensional micro-CT) than treatment with $0.01 \mathrm{mg} / \mathrm{kg}$ or no treatment. Microscopically, these higher doses also reduced inflammatory cell infiltration, bone destruction and positive staining for several proinflammatory cytokines and enzymes in joint tissues.

Although no liver or kidney toxicities were seen in CIA mice treated with bortezomib, nor 